\title{
Feasibility of autologous fibrin glue in general thoracic surgery
}

\author{
Mitsuaki Kawashima ${ }^{1}$, Tadasu Kohno ${ }^{1}$, Sakashi Fujimori ${ }^{1}$, Naoko Kimura ${ }^{1}$, Souichiro Suzuki ${ }^{1}$, \\ Ryuichi Yoshimura $^{1}$, Shinji Yuhara ${ }^{1}$, Akira Kohno ${ }^{1}$, Makoto Wakatabe ${ }^{1}$, Shigeyoshi Makino ${ }^{2}$ \\ ${ }^{1}$ Department of Thoracic Surgery, Respiratory Center, ${ }^{2}$ Department of Transfusion Medicine, Toranomon Hospital, Tokyo, Japan \\ Contributions: (I) Conception and design: M Kawashima, T Kohno, S Makino; (II) Administrative support: T Kohno, S Fujimori, S Makino; (III) \\ Provision of study materials or patients: T Kohno, S Fujimori, S Makino; (IV) Collection and assembly of data: M Kawashima, N Kimura, S Suzuki, \\ R Yoshimura, S Yuhara, A Kohno, M Wakatabe, S Makino; (V) Data analysis and interpretation: M Kawashima, T Kohno, S Fujimori, S Makino; (VI) \\ Manuscript writing: All authors; (VII) Final approval of the manuscript: All authors. \\ Correspondence to: Mitsuaki Kawashima, MD, PhD. Department of Thoracic Surgery, Respiratory Center, Toranomon Hospital, 2-2-2 Toranomon, \\ Minato-ku, Tokyo, 105-8470, Japan. Email: kawashima-gun@umin.ac.jp.
}

Background: Fibrin glue effectively controls air leakage in lung surgery; however, allogenic fibrin glue cannot eliminate the risks of infection and allergy despite current sterilization methods. Autologous fibrin glue (AFG) could be a good alternative, but is not commonly used worldwide because of its limited availability and lack of evidence. Herein, we report clinical outcomes of AFG in thoracic surgery.

Methods: We retrospectively analyzed patients who underwent lobectomies or segmentectomies between November 2016 and September 2017 in our institution. We used two types of AFGs. One was a partiallyautologous fibrin glue (PAFG), the components of which are largely autologous but which contains allogenic thrombin. The other was a completely-autologous fibrin glue (CAFG) which has no allogenic components. PAFG was used in the first half of the study period, after which CAFG was used from March 2017 onward. Patients who did not undergo AFG generation were categorized as the non-AFG group. The perioperative outcomes of the three groups were evaluated.

Results: A total of 207 patients underwent lung surgery, including 118 lobectomies and 89 segmentectomies. Among them, 83 patients received PAFG, 94 received CAFG, and 30 received non-AFG. The mean postoperative drainage period was within a few days in each group (PAFG vs. CAFG vs. nonAFG: $3.23 \pm 3.91$ vs. $3.16 \pm 4.04$ vs. $3.17 \pm 4.16$ days, respectively; $\mathrm{P}=0.405$ ), and the incidence of postoperative prolonged air leakage was within an acceptable range (PAFG vs. CAFG vs. non-AFG: $13.3 \%$ vs. $12.8 \%$ vs. $16.7 \%$, respectively; $\mathrm{P}=0.821$ ).

Conclusions: The use of AFG is clinically feasible for patients who undergo lobectomies or segmentectomies. AFGs could be a viable alternative to conventional allogenic fibrin glues.

Keywords: Fibrin tissue adhesive; lung neoplasms; thoracic surgery; pulmonary surgical procedures

Submitted Jul 08, 2019. Accepted for publication Dec 26, 2019.

doi: $10.21037 /$ jtd.2020.01.01

View this article at: http://dx.doi.org/10.21037/jtd.2020.01.01

\section{Introduction}

Fibrin glue is widely used in various fields including cardiovascular surgery, neurosurgery, and plastic and reconstruction surgery (1). Especially in thoracic surgery, fibrin glue is used not only to control hemorrhage and lymphatic leakage but is very important in managing air leakage, as the patients' lungs are often very fragile because of underlying pulmonary diseases (2). Furthermore, anatomical lung resections require the dissection of the pulmonary hilum and interlobar space, which could cause postoperative air leakage.

The most commonly used fibrin glue is made by allogenic human pooled plasma that comes from multiple donors. Although pasteurization and other infection preventative technologies are routinely performed, there 
are still possibilities of infection with viruses such as human parvovirus (HPV) B19 and hepatitis (3). Moreover, the use of allogenic fibrin glue can lead to anaphylaxis due to the addition of bovine aprotinin (an antifibrinolytic molecule) for clot stabilization (4).

To avoid these risks associated with allogenic fibrin glue, autologous fibrin glue (AFG) may be a viable alternative, and its usage has been minimally reported $(4,5)$. In our hospital, we have mainly used AFG for more than a decade in an attempt to reduce the risks of viral infection and allergic reaction. We previously produced cryoprecipitate from each patient's own plasma by a manual production method and used this cryoprecipitate with allogenic thrombin because of a lack in the technical ability to refine the thrombin component from the patient's plasma. However, the CRYOSEAL system (ASAHI Kasei Medical Co. Ltd., Tokyo, Japan) became available in our hospital in March 2017, which enabled us to prepare the cryoprecipitate and thrombin simultaneously. In this present manuscript, the combination of cryoprecipitate and allogenic thrombin is referred to as partially-autologous fibrin glue (PAFG), while the one generated by CRYOSEAL system as completelyautologous fibrin glue (CAFG).

The purpose of the present study was to investigate the feasibility of AFGs in thoracic surgery. To accomplish this, we surveyed patient demographics and clinical outcomes among those who underwent lung resections using PAFG, CAFG, or non-AFG.

\section{Methods}

Data from patients who underwent lobectomies or segmentectomies in our institute between November 2016 and September 2017 were retrospectively analyzed. Before study commencement, the Institutional Review Board approved our research protocol (approval number: 1670), which was in accordance with the Declaration of Helsinki. Based on its low risk and retrospective nature, the present study received ethical approval for an opt-out methodology regarding patients' participation (6). An accessible overview of our study design is available on our hospital website for patients' reference.

We mainly performed lobectomy plus mediastinal lymph node dissection as a standard procedure for lung cancer. However, there is increasing evidence for the benefit of sublobar resections (7), and so we performed segmentectomies for selected patients who met one of the following criteria: (I) patients with a ground glass opacity nodule with a diameter less than $1.5 \mathrm{~cm}$ and a solid component length of less than half of the tumor diameter; (II) patients with a pulmonary nodule that did not meet criterion (I), but had a pulmonary reserve that could not withstand lobectomy. For patients with metastatic lung cancer, lobectomies or segmentectomies were performed when necessary to obtain adequate surgical margins, as long as any extrapulmonary lesions were well controlled.

\section{Autologous blood storage}

Patients underwent preoperative blood collection unless they had contraindications such as severe anemia (hemoglobin $<11 \mathrm{~g} / \mathrm{dL}$ ), fever, dehydration, active infection, severe heart failure, severe aortic valve stenosis, or other medically unstable conditions. The amount of blood collected for storage was $400 \mathrm{~mL}$ if the patient's body weight (BW) was $50 \mathrm{~kg}$ or greater (8). For patients with a $\mathrm{BW}$ of less than $50 \mathrm{~kg}$, the amount of blood collected was calculated using one of the following formulas: $400 \times \mathrm{BW}$ $(\mathrm{kg}) / 50$, or total blood volume $(\mathrm{TBV}) \times 0.12$. TBV was calculated as described previously (9).

\section{Preparation of PAFG and CAFG}

An essential component of AFG is cryoprecipitate, which is a fraction of plasma, including fibrinogen, growth factors, and various cytokines.

In the PAFG method, cryoprecipitate was prepared from each patient's own plasma, while the thrombin solution was reconstituted from commercially available human thrombin as described previously $(8,9)$. Briefly, each patient's own frozen plasma was thawed at 4 degrees Celsius, and then centrifuged to obtain the cryoprecipitate. Cryo-poor supernatant was collected and restored as frozen plasma for use. This whole process takes approximately 24 to 40 hours (Figure 1A).

In the CAFG method, cryoprecipitate and thrombin were automatically separated from autologous plasma using the CRYOSEAL system as described previously $(10,11)$. Each patient's own frozen plasma was thawed and streamed into the sterile closed CRYOSEAL circuit, then separately filled in packed syringes as cryoprecipitate and thrombin, respectively. This process takes approximately 90 minutes (Figure 1A).

PAFG was made from November 2016 to March 2017, while CAFG was produced from March 2017 to September 2017, as the CRYOSEAL system became 


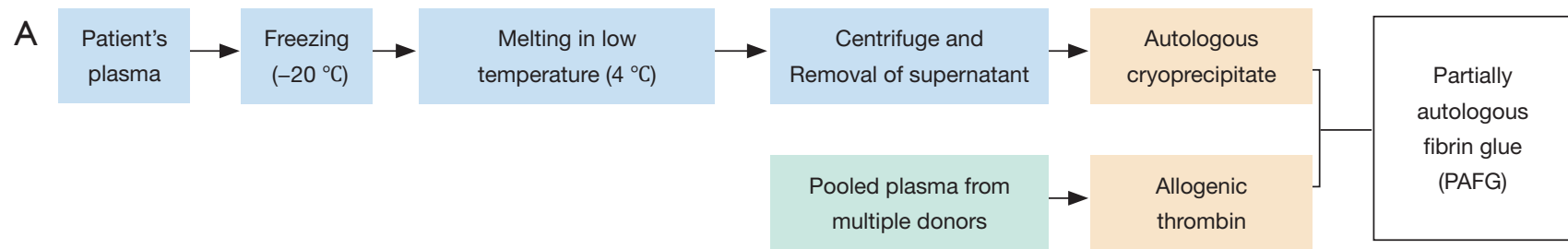

(Hand-made method taking 24 to 40 hours)
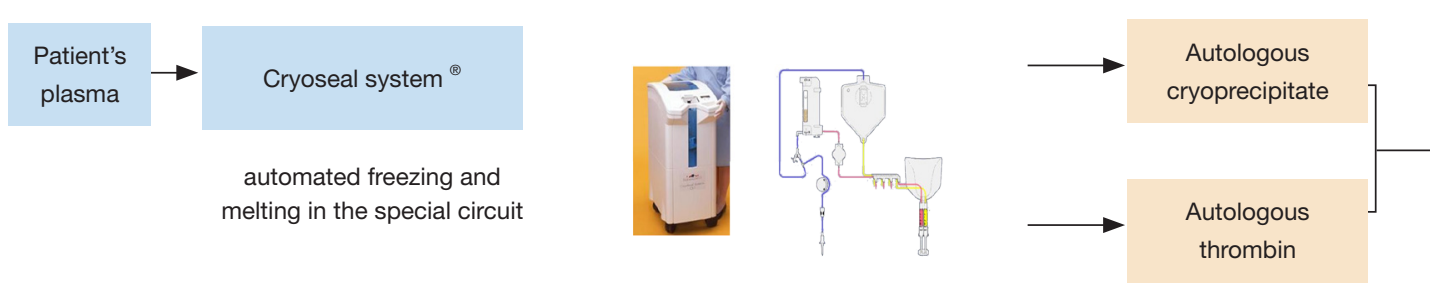

Completely autologous fibrin glue (CAFG)

(Machine-made method taking only 90 minutes)

B

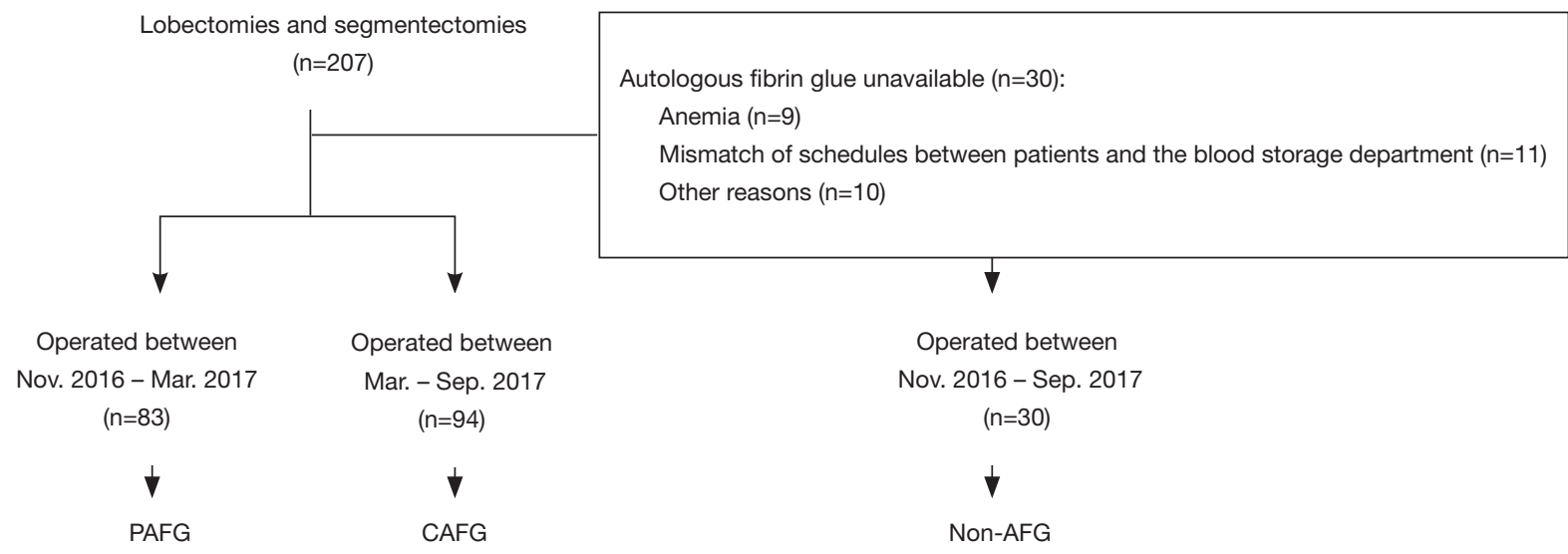

Figure 1 Overview of the study design. (A) The flowcharts indicate the production procedures for PAFG and CAFG; (B) the flowchart depicts the exclusion and patient selection for lung surgery using PAFG or CAFG. Patients who underwent surgery between November 2016 and March 2017 received PAFG, while those who underwent surgery between March and September 2017 received CAFG. Patients who did not undergo AFG generation because of contraindications were assigned to the non-AFG group. PAFG, partially-autologous fibrin glue; CAFG, completely-autologous fibrin glue; AFG, autologous fibrin glue.

available in our hospital in March 2017. Patients who did not undergo AFG generation because of contraindications or mismatch of schedules were assigned to the non-AFG group and received allogenic fibrinogen and thrombin products which were derived from pooled human plasma (Figure 1B).

\section{Surgical procedures and application of fibrin glue}

Most patients (98.6\%) were operated on via threeport video-assisted thoracoscopic surgery, as described previously $(12,13)$. Only three of 207 patients $(1.4 \%)$ underwent open surgery, including chest wall resection (two cases) and pulmonary artery injury (one case). After 
anatomical lung resection plus nodal dissection, major air leakage points were repaired using 4-0 PDS suture (Ethicon, Inc., Cincinnati, OH, USA). One to $2 \mathrm{~mL}$ of fibrin glue was applied to the dissected hilum, lung parenchyma, and sutured points. Several pieces of $1.5 \mathrm{~cm}^{2}$ polyglycolic acid (PGA) sheet (Neoveil sheet, Gunze, Ltd., Tokyo, Japan) were subsequently attached to the points at which the fibrin glue was applied to reinforce the exposed pulmonary parenchyma $(14,15)$. Finally, the remaining fibrin glue was applied to sufficiently cover these areas. One chest tube was placed in all cases, irrespective of the surgical procedure.

\section{Postoperative management}

The amount of air leakage was principally monitored using the Thopaz digital chest drainage system (Medela Healthcare, McHenry, IL, USA), with the pressure set at $-8 \mathrm{cmH}_{2} \mathrm{O}(16)$. However, a conventional drainage system was sometimes applied and used at the water seal setting, whenever Thopaz was not available. A chest tube was removed if air leakage was not detectable for at least 12 hours, and the amount of evacuating fluid was less than $400 \mathrm{~mL} /$ day. Postoperative pleurodesis was performed using OK-432 (Picibanil, Chugai Pharmaceutical Co., Ltd., Tokyo, Japan) in accordance with the surgeons' preferences when air leakage continued for more than several days unless the patient had interstitial lung disease (17). Postoperative prolonged air leakage was defined as sustained air leakage of more than 7 days and/or air leakage of less than 7 days but requiring chemical pleurodesis.

\section{Calculation of medical expenses}

In Japan, the entire population is enrolled in a mandatory health insurance system [Japan National Health Insurance (NHI)] that is organized by the government $(18,19)$. The medical fee is set for all procedures approved by the NHI, and the total in-hospital cost is calculated based on each healthcare service (19). The cost to the patient is usually $30 \%$ of the total cost, with the remainder paid by the NHI; however, the patient cost is reduced to $10 \%$ for older adults (18). These general rules have some exceptions, depending on patients' incomes (18).

The NHI covered lobectomies, segmentectomies, blood storage, and CAFG generation, and the total expenses were calculated for each patient (including other medical services). During the study period, PAFG was not yet covered by the NHI.

\section{Statistical analysis}

The relationships between the clinicopathological features and the type of AFG were assessed using one-way analysis of variance, the Kruskal-Wallis test, Student's $t$-test, MannWhitney U test, or Fisher's exact test. A P value of $<0.05$ was considered statistically significant. Data were analyzed using JMP Pro version 14.0.1 (SAS Institute, Cary, NC, USA).

\section{Results}

A total of 207 consecutive cases were analyzed, including 118 lobectomies and 89 segmentectomies. Of these 207 patients, 83 (40.1\%) received PAFG, 94 (45.4\%) received CAFG, and 30 received non-AFG (14.5\%) (Figure 1B). The clinicopathological features of each group are summarized in Table 1. There were no statistically significant differences among the three groups regarding age, sex, smoking habit, incidences of chronic obstructive pulmonary disease (COPD) or interstitial pneumonia, pathological diagnosis, and surgical procedure (Table 1). However, patients in the non-AFG group trended to be older, and had higher rates of COPD, and interstitial pneumonia compared with the PAFG and CAFG groups (Table 1).

Regarding blood storage, an average of approximately $390 \mathrm{~mL}$ of blood per patient was stored 11 to 13 days prior to an operation (Table 2). Although this procedure caused around a $1.5 \mathrm{mg} / \mathrm{dL}$ drop in hemoglobin before surgery, mean preoperative hemoglobin concentration was $12.5 \mathrm{~g} / \mathrm{dL}$, which was considered an acceptable level for our operation (Table 2). No extra iron supplementation or blood transfusion was required, except in one case with pulmonary artery injury during the surgery. Interestingly, the amount of fibrin glue produced was smaller in the CAFG group than in the PAFG group in spite of there being no difference regarding blood volume storage between the groups (Table 2). No adverse events occurred as a direct result of blood collection. Hemoglobin concentration before blood storage was significantly lower in the non-AFG group, which was attributable to patients with anemia ( $\mathrm{n}=9)$.

Postoperative outcomes were acceptable in each group, including postoperative drainage period of $<3$ days, and low incidences of postoperative prolonged air leakage or chest tube reinsertion (Table 3). There were no postoperative 
Table 1 Patients' demographics

\begin{tabular}{|c|c|c|c|c|}
\hline Variables & PAFG $(n=83)$ & CAFG $(n=94)$ & Non-AFG $(n=30)$ & $P$ value \\
\hline \multicolumn{5}{|l|}{ Sex, n (\%) } \\
\hline Male & $42(50.6)$ & $56(59.6)$ & $18(60.0)$ & \\
\hline Female & $41(49.4)$ & $38(40.4)$ & $12(40.0)$ & $0.459^{b}$ \\
\hline Yes & $43(51.8)$ & $57(60.6)$ & $18(60.0)$ & \\
\hline No & $40(48.2)$ & $37(39.4)$ & $12(40.0)$ & $0.493^{b}$ \\
\hline \multicolumn{5}{|c|}{ Chronic obstructive lung disease, $\mathrm{n}(\%)$} \\
\hline Yes & $9(10.8)$ & $12(12.8)$ & $5(16.7)$ & \\
\hline Yes & $5(6.0)$ & $4(4.3)$ & $5(16.7)$ & \\
\hline No & $78(94.0)$ & $90(95.7)$ & $25(83.3)$ & $0.067^{b}$ \\
\hline \multicolumn{5}{|c|}{ Pathological diagnosis, n (\%) } \\
\hline Primary lung cancer ${ }^{c}$ & $71(85.5)$ & $85(90.4)$ & $23(76.7)$ & \\
\hline Metastatic lung tumor ${ }^{d}$ & $9(10.8)$ & $5(5.3)$ & $5(16.7)$ & \\
\hline Other benign diseases ${ }^{e}$ & $3(3.6)$ & $4(4.3)$ & $2(6.7)$ & $0.252^{b}$ \\
\hline \multicolumn{5}{|l|}{ Surgical procedure, $\mathrm{n}(\%)$} \\
\hline Lobectomy & $48(57.8)$ & $54(57.4)$ & 16 (53.3) & \\
\hline
\end{tabular}

$P$ values determined using the ${ }^{a}$ Kruskal-Wallis test or ${ }^{b}$ Fisher's exact test. ${ }^{c}$, primary lung cancer constituted adenocarcinoma ( $\left.\mathrm{n}=135\right)$, squamous cell carcinoma ( $n=13)$, and other histology $(n=8) ;{ }^{d}$, metastatic lung tumor constituted cancers of the following origins: colon $(n=8)$, kidney $(n=1)$, mammary gland $(n=1)$, hepatobiliary system $(n=1)$, and other organs $(n=3)$; ${ }^{e}$, other benign diseases constituted infectious lung diseases $(n=3)$, organizing pneumoniae $(n=2)$, and miscellaneous $(n=2)$. PAFG, partially-autologous fibrin glue; CAFG, completely-autologous fibrin glue; AFG, autologous fibrin glue; SD, standard deviation.

complications related directly to the use of AFG and nonAFG, such as allergic reaction or viral infection. The inhospital treatment cost was significantly higher in the non-AFG group than in the other groups although the individually-paid medical expenses did not differ statistically between the groups (Table 3).

\section{Discussion}

The present study aimed to evaluate the clinical feasibility of AFG in thoracic surgery. Both PAFG and CAFG achieved acceptable outcomes for patients undergoing lobectomy or segmentectomy. Moreover, preoperative blood storage and AFG preparation were successfully done without any disturbance to following surgical interventions.

Fibrin glue is an essential product in thoracic surgery for controlling air leakage, lymphatic leakage, and bleeding; thus, the availability of allogenic fibrin glue has contributed greatly to achieving surgical safety. Allogenic fibrin glue is generally safe because of sterilization; however, there are still risks of virus transmission and anaphylaxis. HPV B19 is relatively resistant to heat and chemical inactivation, and Kawamura et al. reported that using allogenic fibrin sealant caused transmission of HPV B19, and that the risk was $20 \%$ in patients who were preoperatively negative for antiHPV B19 antibody immunoglobulin G (3). HPV B19 is 
Table 2 Details of blood collection and storage

\begin{tabular}{|c|c|c|c|c|}
\hline Variables & PAFG $(n=83)$ & CAFG $(n=94)$ & Non-AFG $(n=30)$ & $P$ value \\
\hline Amount of autologous fibrin glue (mL) & $5.4 \pm 1.8$ & $4.5 \pm 1.3$ & N/A & $<0.001^{\mathrm{a}}$ \\
\hline Days from blood storage to operation (day) & $11.0 \pm 7.5$ & $12.9 \pm 10.1$ & N/A & $0.563^{\mathrm{a}}$ \\
\hline Hemoglobin concentration before storage $(\mathrm{mL})$ & $13.9 \pm 1.3$ & $14.0 \pm 1.4$ & $12.8 \pm 2.1$ & $0.017^{\mathrm{b}}$ \\
\hline Hemoglobin drop after blood storage $(\mathrm{mL})$ & $1.6 \pm 0.80$ & $1.4 \pm 0.95$ & N/A & $0.267^{\mathrm{b}}$ \\
\hline
\end{tabular}

Data are given as the mean \pm SD. $P$ values determined using ${ }^{a}$ Mann-Whitney $U$ test, ${ }^{b}$ Kruskal-Wallis test, or ${ }^{c} S t u d e n t ' s ~ t$-test. PAFG, partially-autologous fibrin glue; CAFG, completely-autologous fibrin glue; AFG, autologous fibrin glue; N/A, not applicable; SD, standard deviation.

Table 3 Postoperative outcomes

\begin{tabular}{|c|c|c|c|c|}
\hline Variables & PAFG $(n=83)$ & CAFG $(n=94)$ & Non-AFG $(n=30)$ & $P$ value \\
\hline \multicolumn{5}{|l|}{ Postoperative prolonged air leakage $\mathrm{a}^{\mathrm{a}}$} \\
\hline Yes $(\%)$ & $11(13.3)$ & $12(12.8)$ & $5(16.7)$ & \\
\hline No (\%) & $72(86.7)$ & $82(87.2)$ & $25(83.3)$ & $0.821^{\mathrm{C}}$ \\
\hline Yes (\%) & $1(1.2)$ & $2(2.1)$ & $0(0.0)$ & \\
\hline No (\%) & $82(98.8)$ & $92(97.9)$ & $30(100.0)$ & $1.0000^{c}$ \\
\hline Postoperative length of hospital stays (day), mean \pm SD & $6.45 \pm 3.68$ & $7.97 \pm 13.60$ & $7.40 \pm 5.68$ & $0.604^{\mathrm{b}}$ \\
\hline Total in-hospital treatment costs (million JPY), mean \pm SD & $1.72 \pm 0.28$ & $1.81 \pm 0.27$ & $2.27 \pm 1.24$ & $0.016^{\mathrm{b}}$ \\
\hline
\end{tabular}

a common infection, and most people have immunity by adulthood. HPV B19 usually causes minor febrile illness and may be asymptomatic but may cause aplastic crisis (worsening anemia and reticulocytopenia) in patients with chronic hemolytic anemia (20). Regarding anaphylactic reactions, Beierlein et al. reported that bovine aprotinin, an additive in fibrin products, could cause hypersensitivity reactions, and that the risk was $2.8 \%$ in patients with a previous history of exposure to fibrin products (4). Additionally, fatal anaphylaxis related to aprotinin use can occur in patients who are preoperatively positive for antiaprotinin immunoglobulin E (21). The risk of aprotinin sensitization is presumably increasing as populations age, and as more patients are likely to have histories of multiple operations and aprotinin exposure. Considering these risks, AFG could be a good alternative material.

Preoperative blood collections were conducted safely in both the PAFG and CAFG groups. The volumes of blood collected in the PAFG and CAFG groups were sufficient to create fibrin glue (Table 2). The hemoglobin decrease after blood collection was within an acceptable range in both groups, and we did not routinely administer iron supplementation after blood collection. There were no apparent complications regarding blood collection. The duration from blood collection to surgery was less than two weeks in most cases, which is an acceptable waiting time considering other preoperative examinations were simultaneously performed within the same period. These 
data indicate that our blood collection strategy was safe and comparable with previous reports $(8,10,11)$.

The clinical outcomes of lung surgery performed using each of the two types of AFGs were comparable to those in patients in the non-AFG group (Table 3). Interestingly, the resultant amount of fibrin glue was greater in the PAFG group than the CAFG group (Table 2). The productive efficiency of the CRYOSEAL system is reportedly lower than that of the manual method, as CAFG contains less fibrinogen and fibronectin than PAFG (22). Thus, CAFG requires a slightly longer coagulation time (reportedly 3-4 seconds) than PAFG or conventional allogenic glues $(11,23,24)$. However, CAFG has a stronger adhesive force after coagulation $(10,11,24)$. This is because the cryoprecipitate in CAFG is not only composed of fibrinogen and coagulation factor-XIII, but is also rich in coagulation factor-VIII, fibronectin, transforming growth factor- $\beta$, and vascular endothelial growth factor $(10,11,24)$, which are important for wound healing. Hitomi et al. (25) reported better wound healing after the use of CAFG compared with other fibrin glues, probably due to the presence of these growth factors in CAFG. We think that the characteristics of CAFG are clinically acceptable, and may improve postoperative outcomes.

The production of CAFG requires the CRYOSEAL system which is an automatic plasma component separating device. The purchase price of this device is approximately 8 million JPY, while the lease price is 90,000 JPY per month (confirmed on Mar 6th, 2019). However, the introduction of this new device may not be a negative financial burden to the hospital, depending on health insurance systems. Fortunately, the CAFG method was already covered in the Japanese NHI system $(11,24)$, and, thus, resulted in affordable treatment cost (Table 3). During the study period, PAFG was not yet covered by NHI, and we believe that this may be the reason for the cost difference between CAFG and PAFG (Table 3). Recently, PAFG also became covered by the NHI, and the CAFG coverage rate increased from that in 2018 (26); thus, both types of AFGs could become more practical for health care providers in Japan. Of note, caution is warranted when assessing the data in Table 3. We believe the cost differences between the non-AFG and PAFG/CAFG groups is not attributable to differences in the glues because costs of allogenic ones range from $45,000-55,000 \mathrm{JPY}$, which is less than $5 \%$ of total costs. Given patients' older age and higher morbidities (COPD, interstitial pneumonia, and anemia) in the non-AFG group (Tables 1,2), these price differences are presumably secondary to additional care for those comorbidities.

In the field of cardiovascular surgery, one study concluded that the use of the CRYOSEAL system is not routinely recommended, as it did not reduce the transfusion rate despite its high cost (27); however, the design of this previous study is entirely different to that of the present study. Their study used allogenic fibrin glue that was made by the CRYOSEAL system using a blood sample obtained from a single volunteer, not from each patient (27). Furthermore, the surgical procedure in this previous study was coronary artery bypass grafting (including on-pump cases) (27). Considering these differences, we think that the present results should be evaluated independently of this previous report.

Our present study had some limitations. First, the study design was retrospective, observational, nonrandomized, and single-institutional. AFGs were generated only for patients who met the inclusion criteria for blood storage; therefore, patients were not randomly assigned to the AFG or non-AFG groups. However, comparing CAFG and PAFG, there is little bias regarding patient allocation, as we did not change the surgical indication, strategy, basic techniques, or pre- and postoperative patient management during the study period. Second, an effect of using PGA sheets might have overshadowed the effectiveness of AFG. Although this situation is not ideal for evaluating the role and impact of AFG itself, we believe that using AFG is acceptable and safe, at least combined with PGA sheets in thoracic surgery.

In conclusion, both types of AFG are clinically feasible and showed satisfactory outcomes for patients who undergo lobectomies or segmentectomies. AFG could be a viable alternative to conventional allogenic fibrin glues. Further studies are desired to validate their effectiveness.

\section{Acknowledgments}

We thank Tetsufumi Futatsugi (Medical Affairs Section, Toranomon Hospital) for his help in calculating the inhospital charges. We appreciate ASAHI Kasei Medical Co. Ltd. for generous permission to reproduce their marketing materials in the $1^{\text {st }}$ illustration. We thank Dr. Kelly Zammit, BVSc, and Dr. Jane Charbonneau, DVM, from Edanz Group (www.edanzediting.com/ac), for editing a draft of this manuscript. 
Funding: None.

\section{Footnote}

Conflicts of Interest: The authors have no conflicts of interest to declare.

Etbical Statement: The authors are accountable for all aspects of the work in ensuring that questions related to the accuracy or integrity of any part of the work are appropriately investigated and resolved. This study was approved by the Institutional Review Board in Toranomon Hospital (approval number: 1670).

Open Access Statement: This is an Open Access article distributed in accordance with the Creative Commons Attribution-NonCommercial-NoDerivs 4.0 International License (CC BY-NC-ND 4.0), which permits the noncommercial replication and distribution of the article with the strict proviso that no changes or edits are made and the original work is properly cited (including links to both the formal publication through the relevant DOI and the license). See: https://creativecommons.org/licenses/by-nc-nd/4.0/.

\section{References}

1. Jackson MR. Fibrin sealants in surgical practice: An overview. Am J Surg 2001;182:1S-7S.

2. Fabian T, Federico JA, Ponn RB. Fibrin glue in pulmonary resection: a prospective, randomized, blinded study. Ann Thorac Surg 2003;75:1587-92.

3. Kawamura M, Sawafuji M, Watanabe M, et al. Frequency of Transmission of Human Parvovirus B19 Infection by Fibrin Sealant Used During Thoracic Surgery. Ann Thorac Surg 2002;73:1098-100.

4. Beierlein W, Scheule AM, Dietrich W, et al. Forty years of clinical aprotinin use: a review of 124 hypersensitivity reactions. Ann Thorac Surg 2005;79:741-8.

5. Kjaergard HK, Weis-Fogh US, Sørensen H, et al. Autologous fibrin glue--preparation and clinical use in thoracic surgery. Eur J Cardiothorac Surg 1992;6:52-4; discussion 54.

6. Vellinga A, Cormican M, Hanahoe B, et al. Opt-out as an acceptable method of obtaining consent in medical research: a short report. BMC Med Res Methodol 2011;11:40.

7. Nakazawa S, Shimizu K, Mogi A, et al. VATS segmentectomy: past, present, and future. Gen Thorac
Cardiovasc Surg 2018;66:81-90.

8. Kikuchi D, Iizuka T, Nomura K, et al. Feasibility of Autologous Fibrin Glue and Polyglycolic Acid Sheets to Prevent Delayed Bleeding after Endoscopic Submucosal Dissection of Gastric Neoplasms in Patients Receiving Antithrombotic Therapy. Gastroenterology Research and Practice 2018;2018:2174957.

9. Neyrinck $M$, Vrielink H. Apheresis training for nurses and physicians around the World. J Clin Apher 2015;30:32-7.

10. Kouketsu A, Nogami S, Yamada-Fujiwara M, et al. Clinical evaluations of complete autologous fibrin glue, produced by the CryoSeal ${ }^{\circledR}$ FS system, and polyglycolic acid sheets as wound coverings after oral surgery. J Craniomaxillofac Surg 2017;45:1458-63.

11. Nakayama N, Yano H, Egashira Y, et al. Efficacy, Reliability, and Safety of Completely Autologous Fibrin Glue in Neurosurgical Procedures: Single-Center Retrospective Large-Number Case Study. World Neurosurg 2018;109:e819-e828.

12. Mun M, Kohno T. Efficacy of thoracoscopic resection for multifocal bronchioloalveolar carcinoma showing pure ground-glass opacities of $20 \mathrm{~mm}$ or less in diameter. J Thorac Cardiovasc Surg 2007;134:877-82.

13. Kohno T, Fujimori S, Kishi K, et al. Safe and effective minimally invasive approaches for small ground glass opacity. Ann Thorac Surg 2010;89:S2114-7.

14. Itano $\mathrm{H}$. The optimal technique for combined application of fibrin sealant and bioabsorbable felt against alveolar air leakage. Eur J Cardiothorac Surg 2008;33:457-60.

15. Nakamura T, Suzuki K, Mochizuki T, et al. An evaluation of the surgical morbidity of polyglycolic acid felt in pulmonary resections. Surg Today 2010;40:734-7.

16. De Waele M, Agzarian J, Hanna WC, et al. Does the usage of digital chest drainage systems reduce pleural inflammation and volume of pleural effusion following oncologic pulmonary resection?-A prospective randomized trial. J Thorac Dis 2017;9:1598-606.

17. Ogawa K, Takahashi Y, Murase K, et al. OK-432 pleurodesis for the treatment of pneumothorax in patients with interstitial pneumonia. Respir Investig 2018;56:410-7.

18. Ikegami N, Yoo BK, Hashimoto H, et al. Japanese universal health coverage: evolution, achievements, and challenges. Lancet 2011;378:1106-15.

19. Kajiwara N, Patrick Barron J, Kato Y, et al. CostBenefit Performance of Robotic Surgery Compared with Video-Assisted Thoracoscopic Surgery under the Japanese National Health Insurance System. Ann Thorac 
Cardiovasc Surg 2015;21:95-101.

20. Hino M, Ishiko O, Honda KI, et al. Transmission of symptomatic parvovirus B19 infection by fibrin sealant used during surgery. Br J Haematol 2000;108:194-5.

21. Oswald AM, Joly LM, Gury C, et al. Fatal intraoperative anaphylaxis related to aprotinin after local application of fibrin glue. Anesthesiology 2003;99:762-3.

22. Buchta C, Dettke M, Funovics PT, et al. Fibrin sealant produced by the CryoSeal FS System: product chemistry, material properties and possible preparation in the autologous preoperative setting. Vox Sang 2004;86:257-62 .

23. Shimizu M, Wakimoto N, Tsurumaru M, et al. Clinical evaluation of the use of novel and completely autologous fibrin glue during surgical procedures: Prospective open multicenter trial of the CryoSeal FS System. Jpn J Transfus Cell Ther 2009;55:604-10.

24. Makino S, Kanemura N, Fujishima N, et al. Standards for

Cite this article as: Kawashima M, Kohno T, Fujimori S, Kimura N, Suzuki S, Yoshimura R, Yuhara S, Kohno A, Wakatabe M, Makino S. Feasibility of autologous fibrin glue in general thoracic surgery. J Thorac Dis 2020;12(3):484-492. doi: $10.21037 /$ jtd.2020.01.01 the automatic preparation and use of autologous fibrin glue (article in Japanese). Jpn Soc Autolog Blood Transfus 2014;27:153-62.

25. Hitomi A, Futono K, Hirasawa Y, et al. Promotive effects of autologous fibrin glue "CryoSeal" on wound healing - comparison of the effects with commercially available fibrin glues (article in Japanese). Jpn Pharmacol Ther 2012;40:421-6.

26. The Japan Society of Transfusion Medicine and Cell Therapy. Medical Fees. Accessed 07 Jul 2019. Available online: http://yuketsu.jstmct.or.jp/medical/medicine_and_ medical_information/fee/

27. Tavilla G, Bruggemans EF, Gielen CL, et al. Multicentre randomized clinical trial to investigate the costeffectiveness of an allogeneic single-donor fibrin sealant after coronary artery bypass grafting (FIBER Study). Br J Surg 2015;102:1338-47. 